\title{
Predicting of Nursing Student's Eating Disorder Based on Alexithymia, Coping Styles and Cognitive Emotion Regulation
}

\author{
Azita Amirfakhraei ${ }^{1}$, Sana Rezaei ${ }^{2}$, Zahra DashtBozorgi ${ }^{3},{ }^{*}$, iD
}

\author{
${ }^{1}$ Assistant Professor, Department of Psychology, Young Research and Elite Club, BandarAbbas Branch, Islamic \\ Azad University, BandarAbbas, Iran \\ ${ }^{2}$ MA of Personality Psychology, Department of Psychology, Ahvaz Branch, Islamic Azad University, Ahvaz, \\ Iran \\ ${ }^{3}$ Assistant Professor, Department of Psychology, Ahvaz Branch, Islamic Azad University, Ahvaz, Iran \\ * Corresponding author: Zahra DashtBozorgi, Assistant Professor, Department of Psychology, Ahvaz Branch, \\ Islamic Azad University, Ahvaz, Iran. E-mail: zahradb2000@yahoo.com
}

Received: 29 Dec 2017

Accepted: 06 Nov 2018

\begin{abstract}
Introduction: In recent years, the eating disorder as a psychosocial disorder has increased dramatically and this disorder has an important role in reduction of physical and mental health. This research aimed to predicting of nursing students' eating disorder based on alexithymia, coping styles and cognitive emotion regulation.
\end{abstract}

Methods: This study was a descriptive from correlational type. The research population was the nursing students' of Islamic Azad University of Ahvaz branch in 2017-18 years which from them 210 student (29 man and 181 woman) were selected by stratified randomly sampling method. All of them completed the questionnaires of eating disorder, alexithymia, coping styles and cognitive emotion regulation. Data was analyzed with using SPSS-21 and by Pearson correlation and multiple regression with enter model methods. Results: The findings showed that alexithymia $(r=0.173)$, avoid-focused coping style $(r=0.198)$ and negative cognitive emotion regulation strategies $(r=0.465)$ had a significantly positive relationship with nursing students' eating disorder and problem-focused coping style $(r=-0.376)$ and positive cognitive emotion regulation strategies $(r=-0.541)$ had a significantly negative relationship with nursing students' eating disorder. Also, the variables of positive cognitive emotion regulation strategies, negative cognitive emotion regulation strategies and problem-focused coping style significantly could predict 45.5 percent of variance of nursing students' eating disorder that in this prediction the share of positive cognitive emotion regulation strategies was higher than other variables $(\mathrm{P}<0.05)$.

Conclusions: The results indicate the role and importance of the variables of positive and negative cognitive emotion regulation strategies and problem-focused coping style in predicting nursing students' eating disorder. Therefore counselors and therapists should pay attention to the signs of mentioned variables and based on design and implement appropriate programs to decrease the nursing student's eating disorder.

Keywords: Eating Disorder, Nursing Students, Alexithymia, Coping Styles, Cognitive Emotion Regulation (c) 2018 Iranian Nursing Scientific Association (INA) 


\title{
ييشبينى اختلال خوردن دانشجويان يرستارى بر اساس ناكويى هيجانى، سبكهاى مقابلهاى و تنظيم شناختى هيجان
}

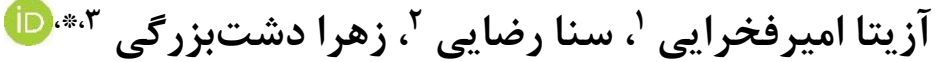

' استاديار، كروه روانشناسى، باشخاه يزووهشكران جوان و نخبحًان، واحد بندرعباس، دانشخاه آزاد اسلامى، بندرعباس، ايران

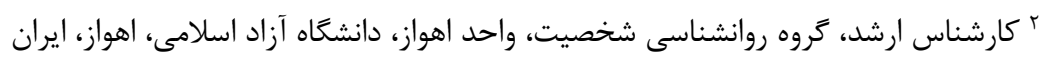

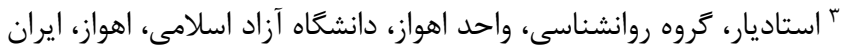

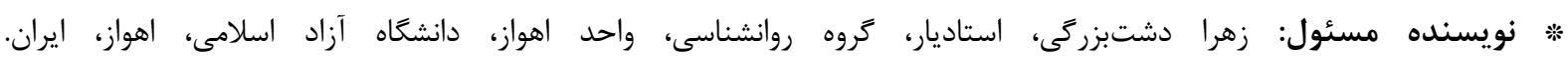
ايميل:zahradb2000@yahoo.com

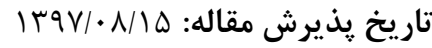

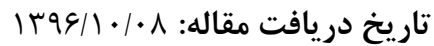

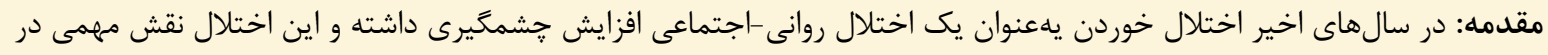

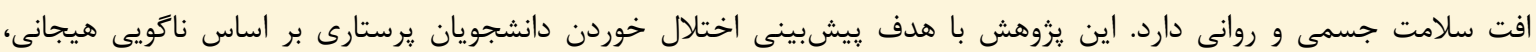

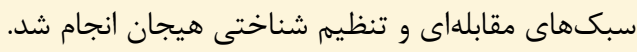

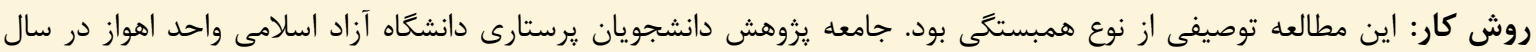

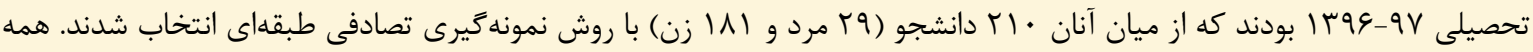

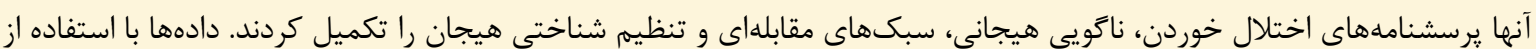

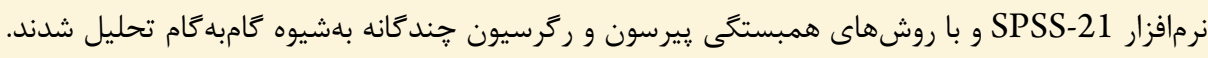

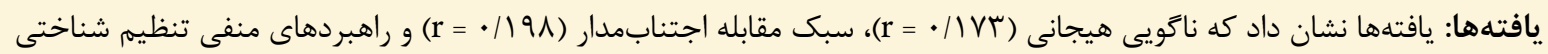

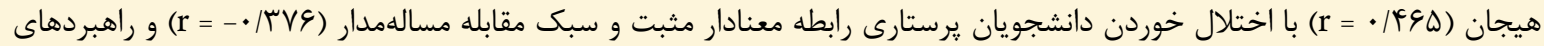

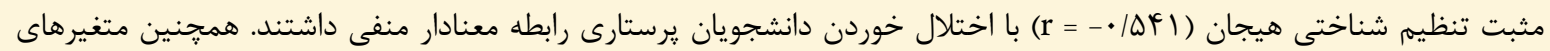

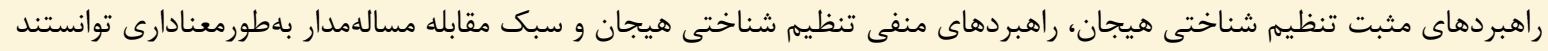

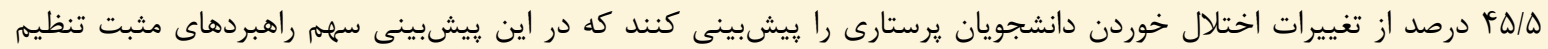

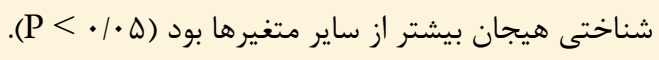

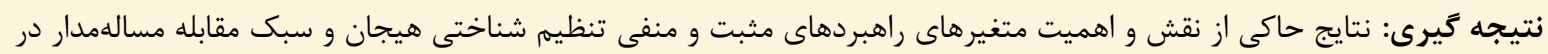

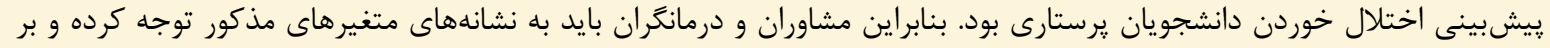

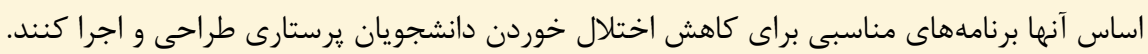

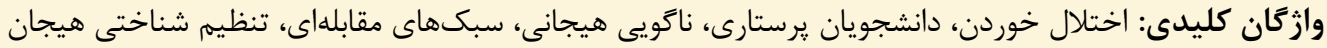

رفتارها و نكَرشهاى نادرست خوردن است كه بهصورت يك زنجيره

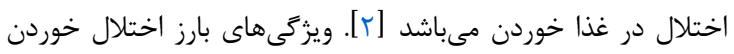

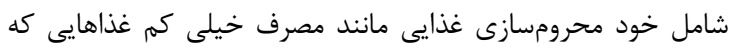

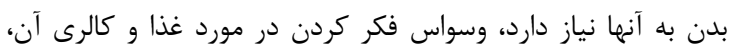

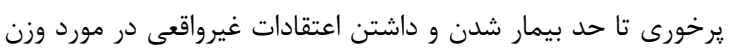

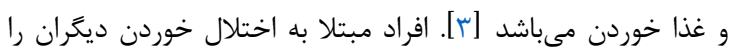

محيط كارى يرستاران به دليل فشارها و استرسهاى شغلى زياد بر

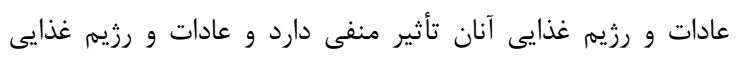

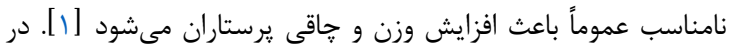

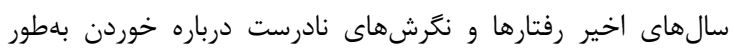

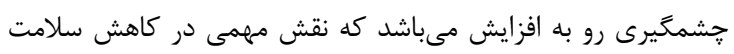

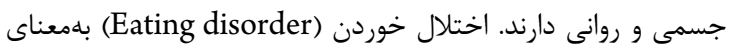


كاهش دهد. سبكهاى اجتنابمدار راهبردهايى را دربرمى گيرند كه در

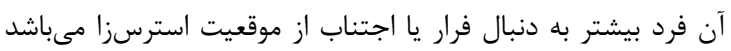

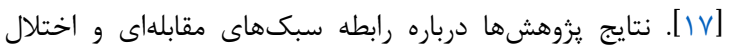

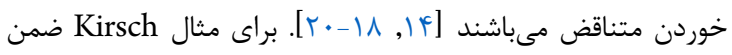
يزوهشى درباره نقش ميانجى سبكهاى مقابلهاى در رابطه بين تصوير

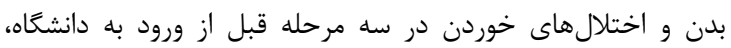

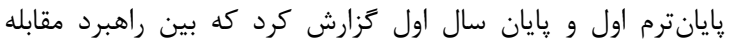

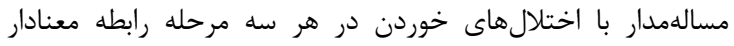

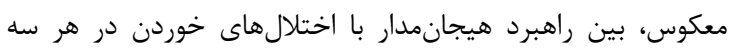

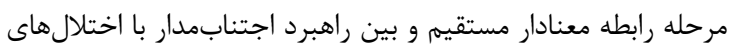

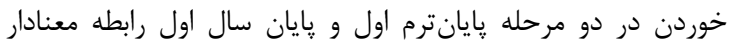

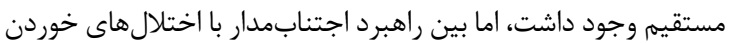

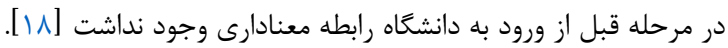

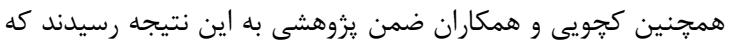

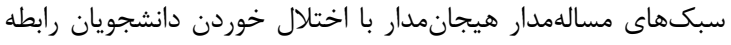
مثبت و معنادار داشتند [9 [1]. در يزوهشى ديكار ديخر اصغرى و و همكاران

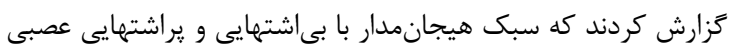

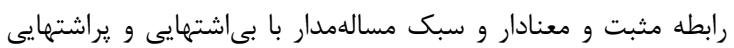
عصبى رابطه منفى و معنادار داشت [ • [r].

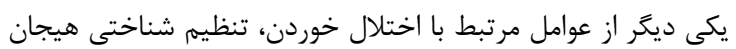
(Cognitive Emotion Regulation) به اعمالى اطلاق مىشود كه بهمنظور تغيير يا تعديل تجربه هيجانى، مئل

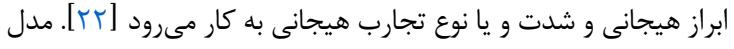

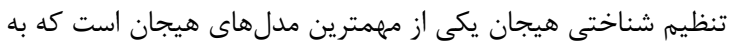

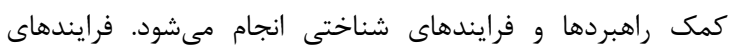

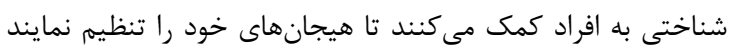

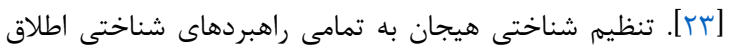

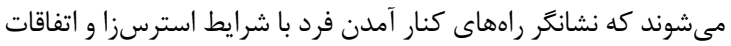

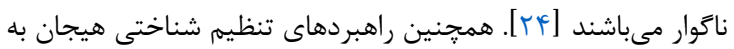

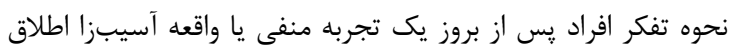

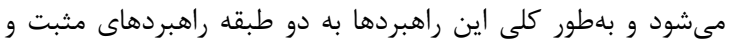

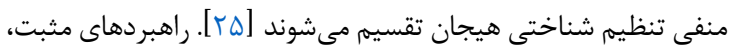

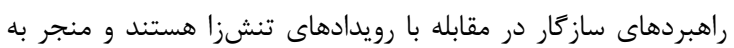

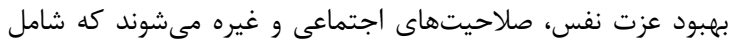

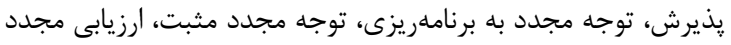

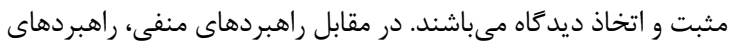

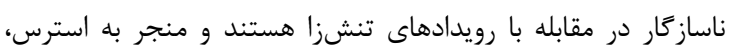

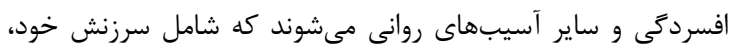

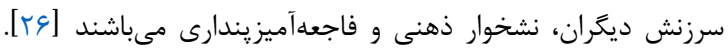

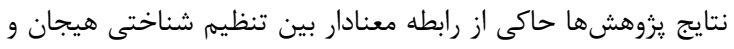

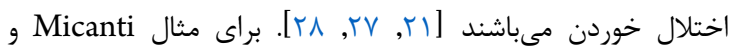
همكاران ضمن يروهشى كزارش كردند كه بين مشكلات تنظيم هيجان

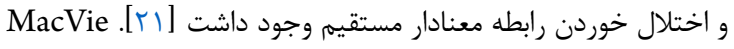
ضمن يزوهشى درباره رابطه راهبردهاى تنظيم هيجان و علائم اختلال

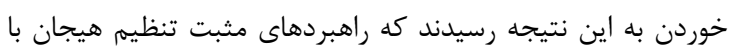

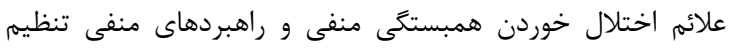

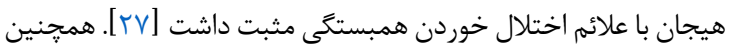

بلهورت سلسله مراتبى درجهبندى مى كنند. به عبارت ديخر آنان افراد

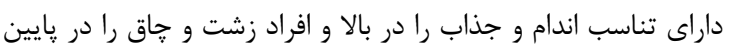

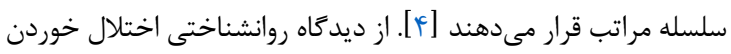

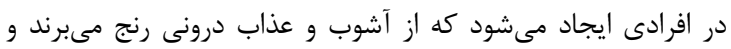

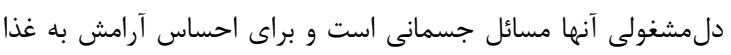

رومىآورند [ها].

اختلال خوردن داراى خصوصيات بارزى از جمله مختل شدن رفار رفتارهاى

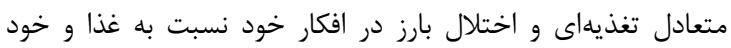
مىباشد كه معمولترين آنها هرزهخوارى، اختلال نشخوار، درئ اختلال

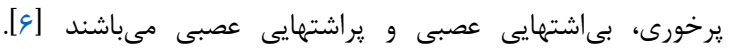

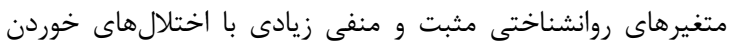

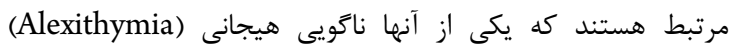

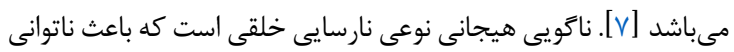

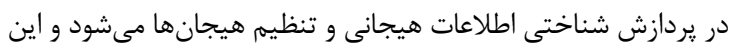

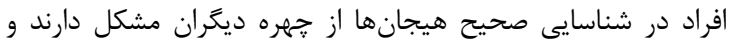

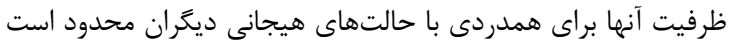

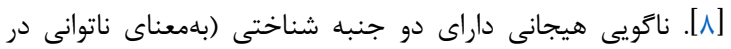

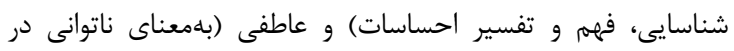

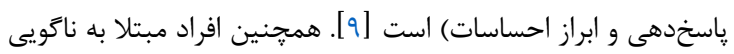

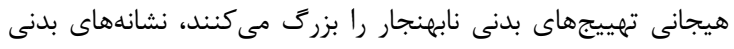

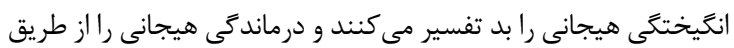

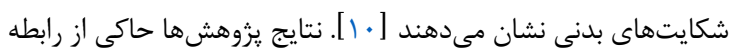

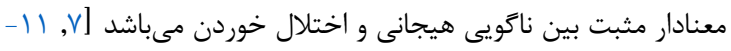

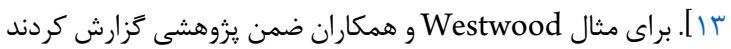

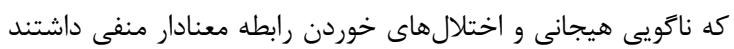

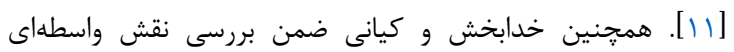

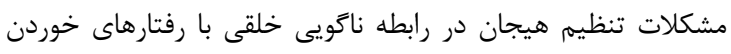

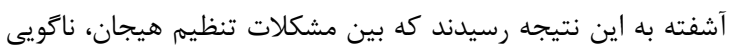

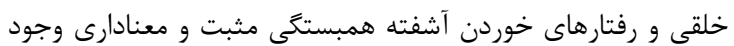
داشت [r/]]. يكى ديخر از عوامل مرتبط با اختلال خوردن، سبكهاى مقابلهاى (Coping styles)

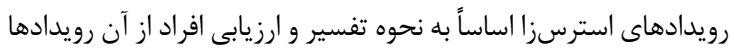

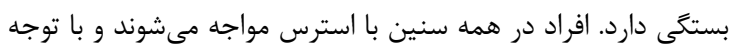

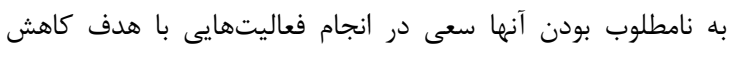

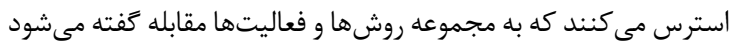

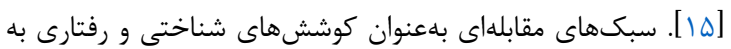
منظور افزايش كنترل و اداره موقعيتهاى تنشزا، تطابق فرد با محيط

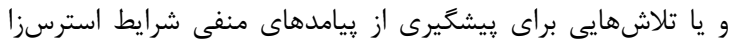

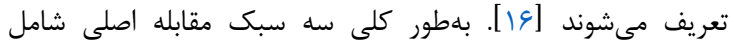

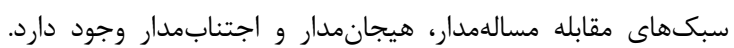

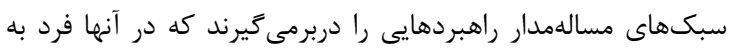

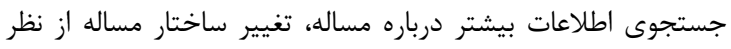

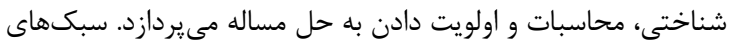

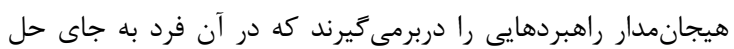

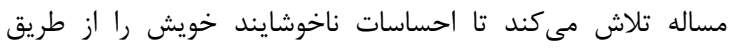

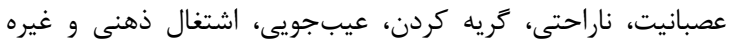


خوردن، ناگويى هيجانى، سبكهاى مقابلهاى و تنظيم شناختى هيجان را تكميل كردند.

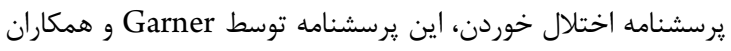

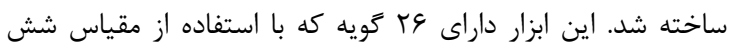

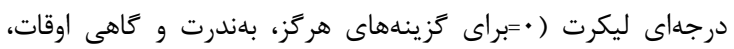

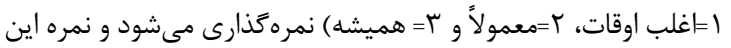

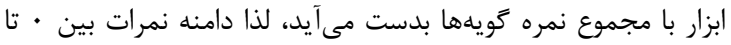

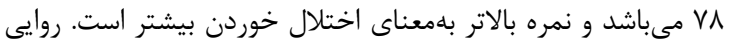

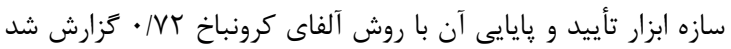

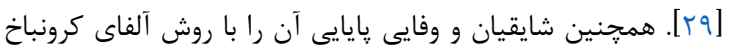

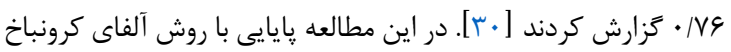

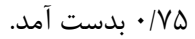

يرسشنامه ناگويى خلقى تورنتو: يرسشنامه ناگويى خلقى

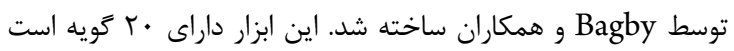

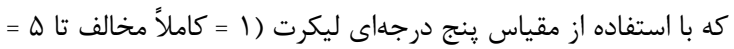

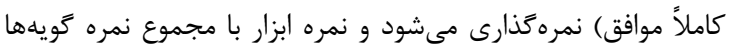

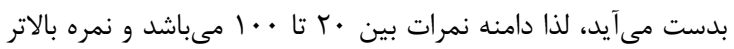

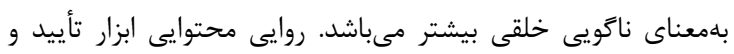

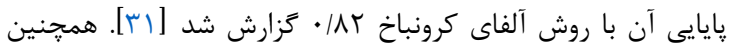

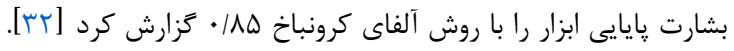

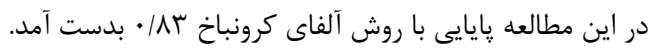

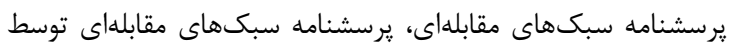
Endler \& Parker

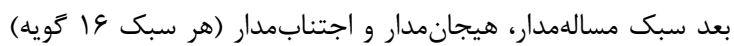

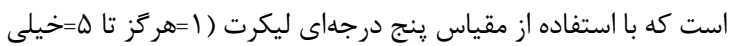

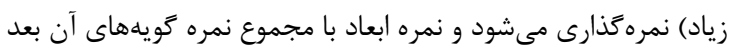

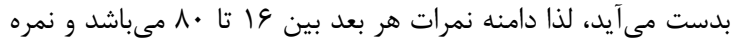

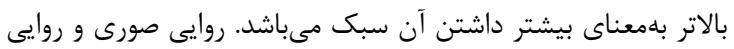

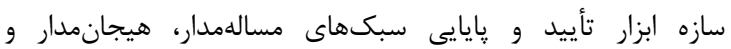

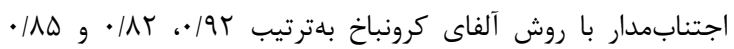

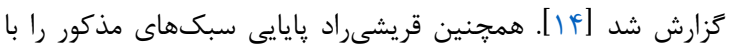

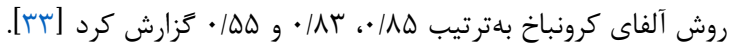

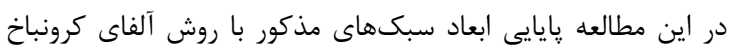

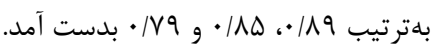

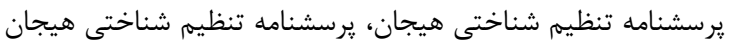

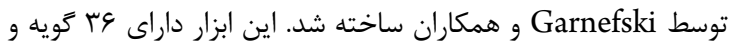

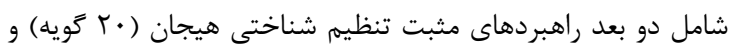
راهبردهاى منفى تنظيم شناختى هيجان (19 كويه) است كه با استفاده

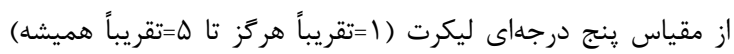

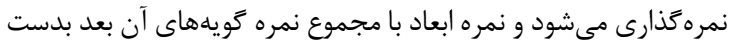

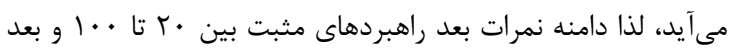

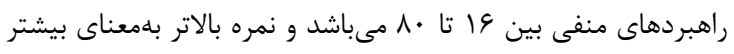

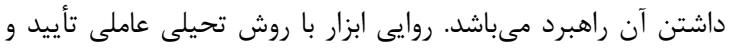

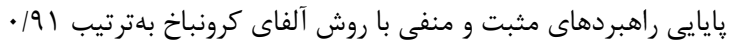

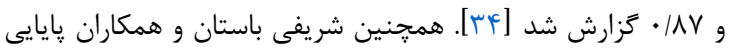

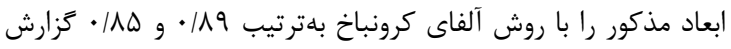

فيروزى ضمن يزوهشى كزارش كرد كه سبكهاى دلبستخى نايمن،

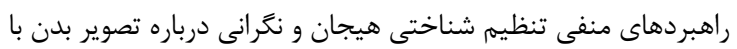

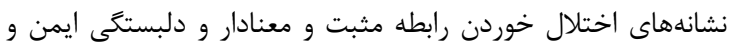

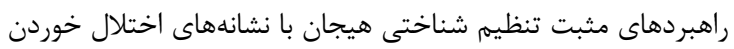

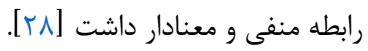

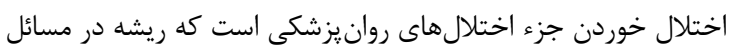

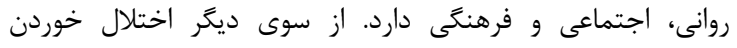

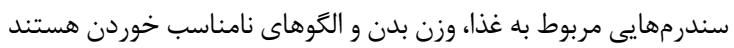

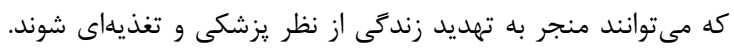

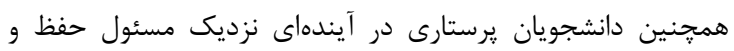

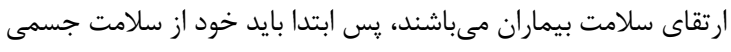

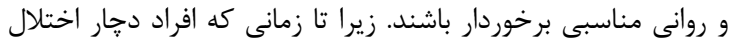

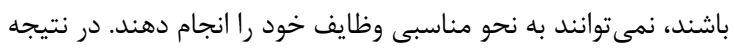

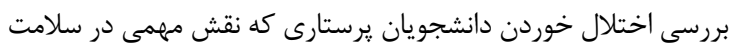

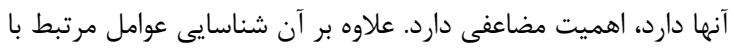

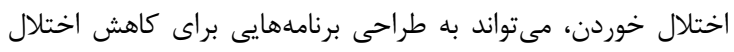

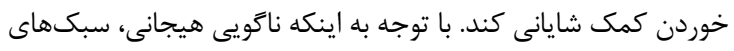

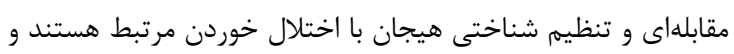

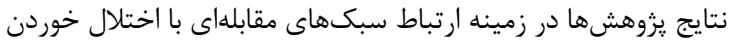

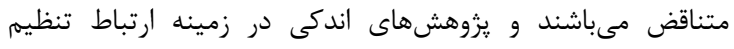

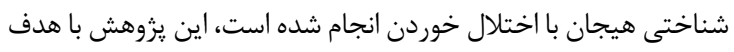

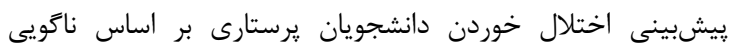

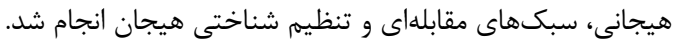

\section{روش كار}

طرح يزوهش توصيفى از نوع همبستخى بود. جامعه يُزوهش دانشجويان

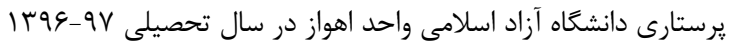

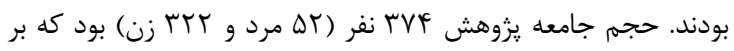

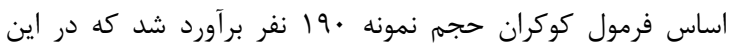

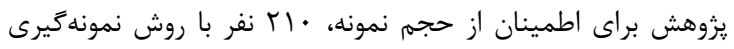

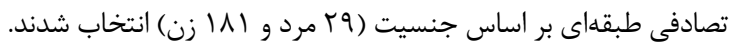

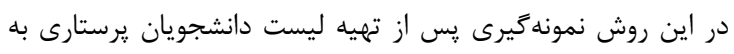

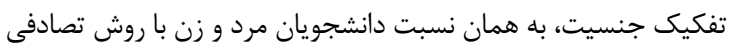

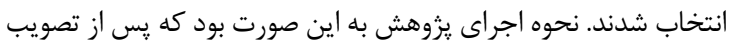

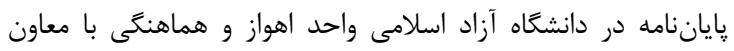

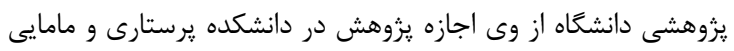

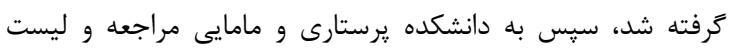

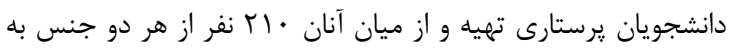

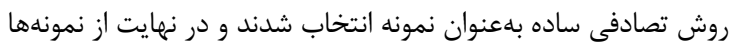

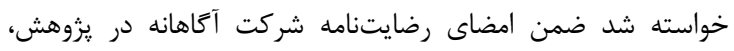

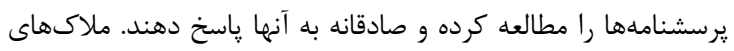

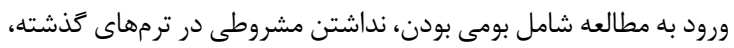

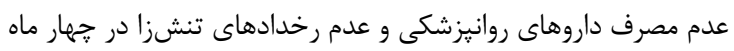

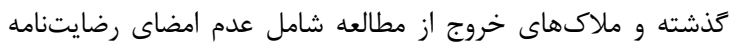

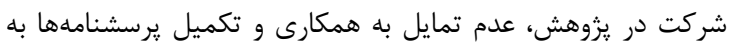

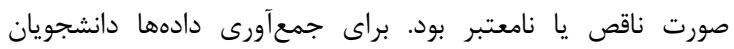

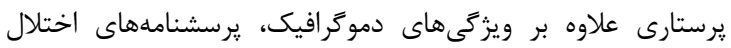


و در سطح استنباطى از روشهاى همبستخى يِيرسون و ركرسيون

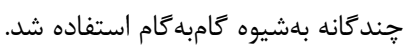

\section{افتهنها}

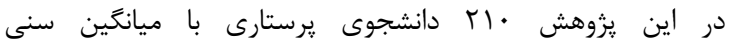

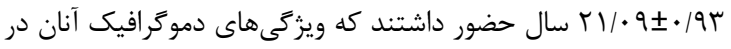

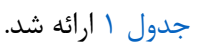

كردند [مَّ]. در اين مطالعه پايايى ابعاد مذكور با روش آلفاى كرونباخ

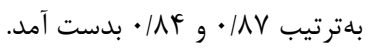

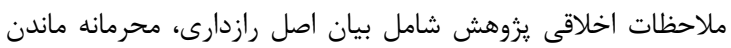

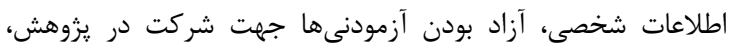

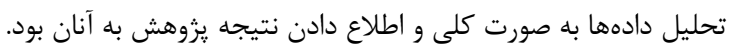

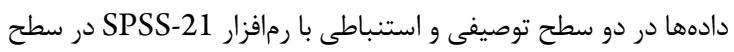

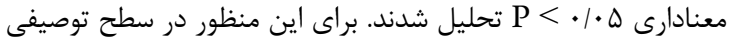

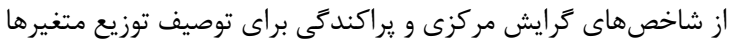

جدول ا: ويزّكىهاى دموگرافيك دانشجويان برستارى به تفكيك وضعيتهاى جنسيت، تأهل و سال تحصيلى

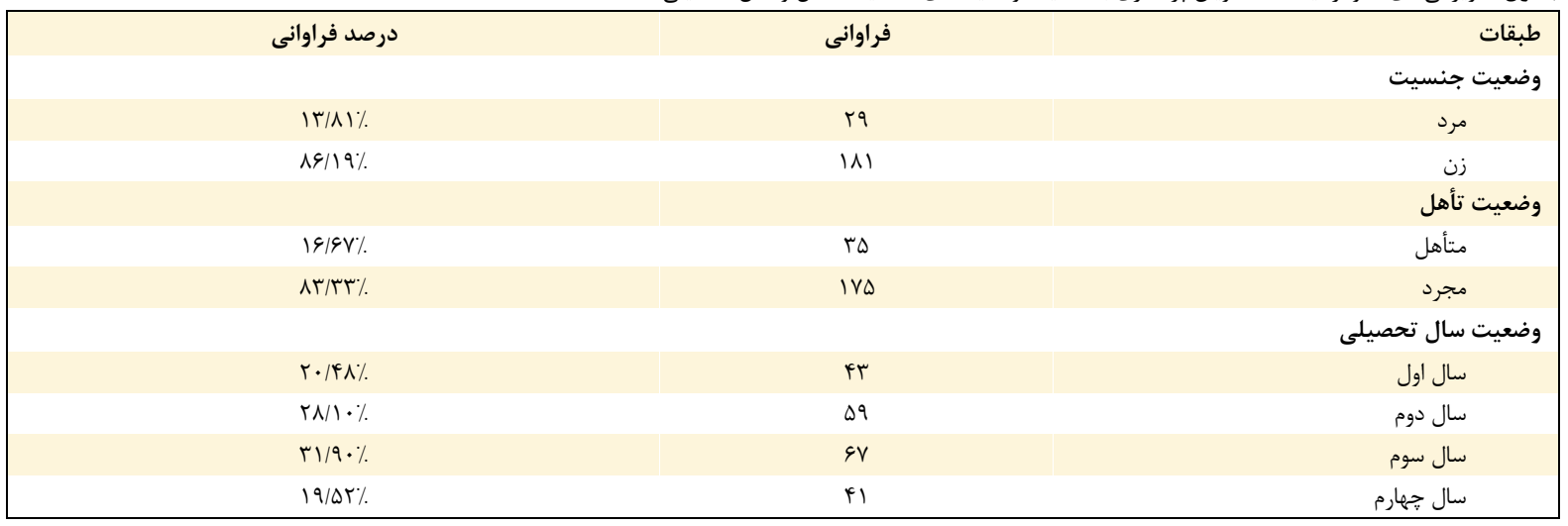

مقدار متغيرهاى ناًويى هيجانى، سبكهاى مقابلهاى، تنظيم شناختى هيجان و اختلال خوردن در جدول بال ارائه شد.

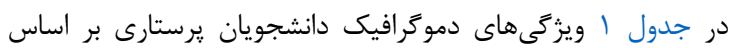

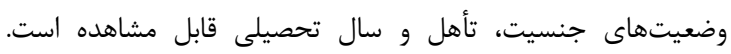
شاخصهاى توصيفى ميانگين، انحراف معيار، كمترين مقدار و بيشترين

\begin{tabular}{|c|c|c|c|c|}
\hline بيشترين مقدار & كمترين مقدار & انحراف معيار & ميانكين & متغير هاى بزوهش \\
\hline v9 & tr & g|Av| & FF/DG & ناكويى هيجانى \\
\hline ve & rA & $1 / 19$ & DT/AFV & سبك مسالهمدار \\
\hline 90 & r & $\Delta / I V$ & $r 9 / 990$ & سبك هيجانمدار \\
\hline vi & r & G/VDI & Fr/fqR & سبك اجتنابمدار \\
\hline 94 & $r \Lambda$ & $9 / T Y$. & SV/DTI & راهبردهاى مثبت تنظيم شناختى هيجان \\
\hline VF & ro & $\mathrm{V} / \mathrm{TIT}$ & $f \mid / \varepsilon \cdot V$ & راهبردهاى منفى تنظيم شناختى هيجان \\
\hline$\Delta V$ & r & $9 / 194$ & rq/rTr & اختلال خوردن \\
\hline
\end{tabular}

تنظيم شناختى هيجان و اختلال خوردن دانشجويان يرستارى از ضريب

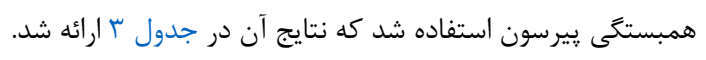

در جدول r شاخصهاى توصيفى متغيرهاى ناخويى هيجانى، سبكهاى

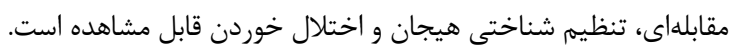

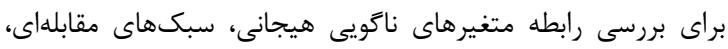

\begin{tabular}{|c|c|c|c|c|c|c|}
\hline \multirow[t]{6}{*}{9} & $\Delta$ & F & $r$ & $r$ & 1 & 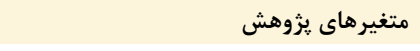 \\
\hline & & & & & 1 & 1. ناكويى هيجانى \\
\hline & & & & 1 & $-\cdot|\mathcal{F V}|^{\text {糟 }}$ & r. سبك مسالهمدار \\
\hline & & & 1 & $-\cdot / \cdot 9 \vee$ & $\cdot / r \Lambda Y^{* * *}$ & r. سبك هيجانمدار \\
\hline & & 1 & $.1 .9 V$ & $-\cdot / r \mid Q^{*}$ & $\cdot / K 99^{* * 3}$ & F. سبك اجتنابمدار \\
\hline & 1 & $-\cdot / 1 \Delta T$ & -.1119 & $\cdot / 191^{*}$ & $-\cdot / \mathbb{R} \Delta^{* * *}$ & ه. راهبردهاى مثبت تنظيم شناختى هيجان \\
\hline 1 & $-\cdot / \Gamma^{G} \cdot{ }^{*}$ & $\cdot / T \mid K^{* * *}$ & $\cdot / 1 \xi^{*}=$ & $-\cdot / 11 f$ & $\cdot / r q \varphi^{* * *}$ & 9. راهبردهاى منفى تنظيم شناختى هيجان \\
\hline$\cdot / \uparrow \& D^{* * *}$ & 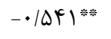 & $\cdot / 19 \Lambda^{*}$ &.$/ 1 r t$ & $-\cdot / r V \varphi^{* *: *}$ & $\cdot / / \mathrm{V}^{\frac{*}{*}}$ & V. اختلال خوردن \\
\hline
\end{tabular}




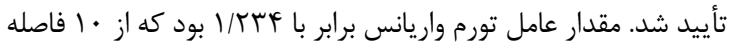

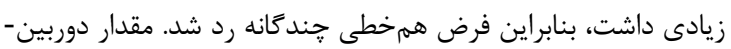

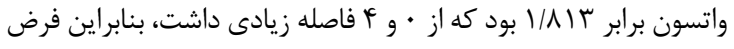

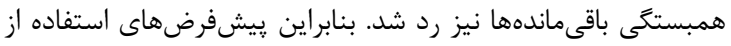

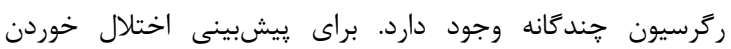

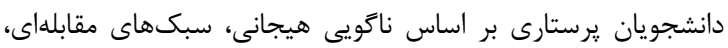

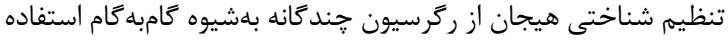
شد كه نتايج آن در جدول \&

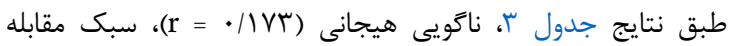

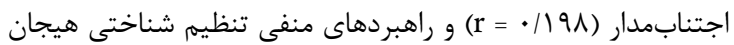

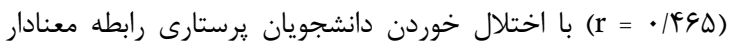

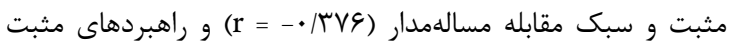

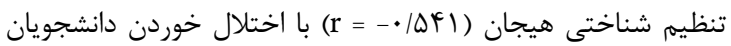

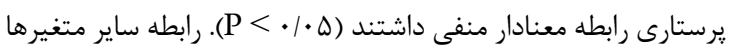

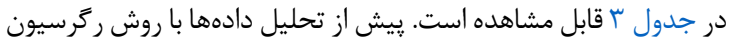

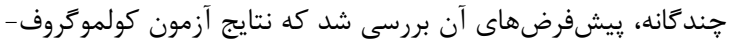

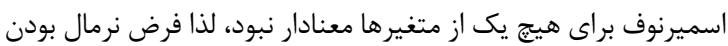

جدول F: نتايج تحليل ركرسيون حندكانه بهشيوه كامبه كام براى بيشبينى اختلال خوردن دانشجويان يرستارى

\begin{tabular}{|c|c|c|c|c|c|c|c|c|}
\hline 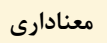 & df2 & df1 & F تغيير F & تغيير Re & $\mathbf{R}^{2}$ & $\mathbf{R}$ & متغير هاى بيشبين & مدل \\
\hline$\cdot 1 \cdot 1$ & $r \cdot \Lambda$ & 1 & $94 / V \cdot 1$ & - rat & . rat & $\cdot|\Delta F|$ & راهبردهاى مثبت تنظيم شناختى هيجان & 1 \\
\hline$\cdot \cdot \cdot 1$ & $r \cdot v$ & 1 & 10/ & $\cdot / 1 \cdot 4$ & $\cdot / 49 \Delta$ & .1949 & راهبردهاى مثبت و منفى تنظيم شناختى هيجان & $r$ \\
\hline.$\cdot \cdot r$ & r.9 & 1 & $11 / A r t$ & .1 .4 & $\cdot / 4 \Delta \Delta$ & $.19 \vee \Delta$ & راهبردهاى مثبت و منفى تنظيم شناختى هيجان و سبك مقابله مسالهمدار & $r$ \\
\hline
\end{tabular}

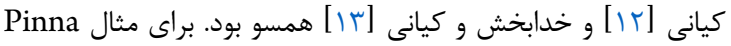

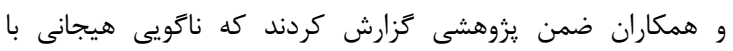

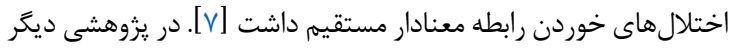

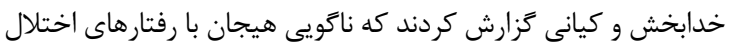

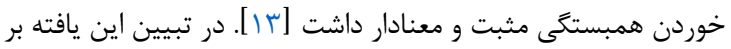

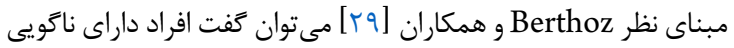

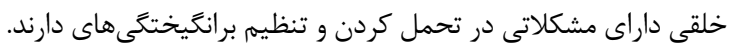

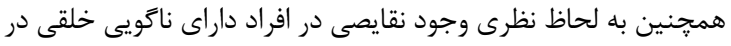

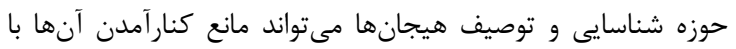

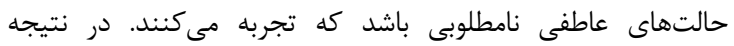
رفتارهاى خوردن بهعنوان كاركرد تنظيم عواطف به آنها كمك مي كئ كند.

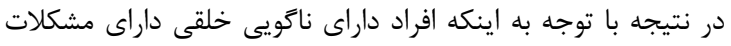

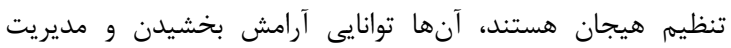

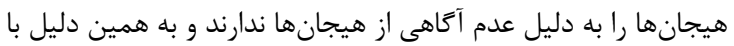

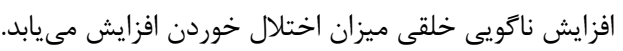

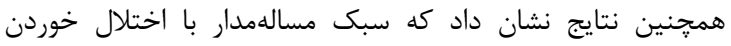
دانشجويان يرستارى رابطه معنادار منفى و سبك هيجانمدار بار با اختلال

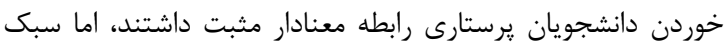

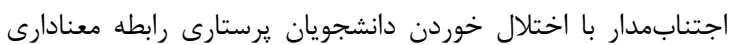

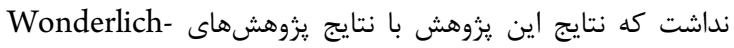

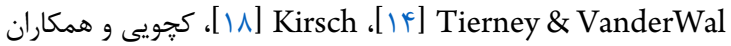

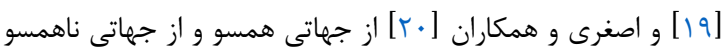

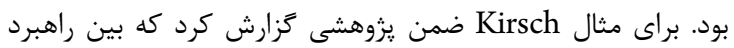

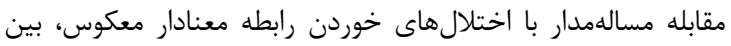

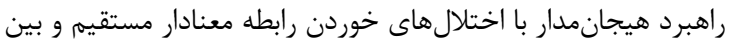

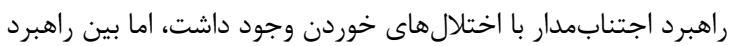

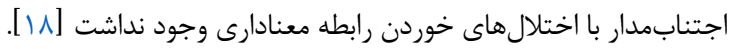

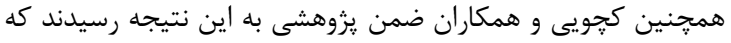

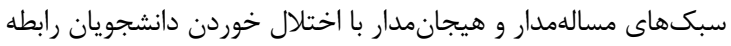

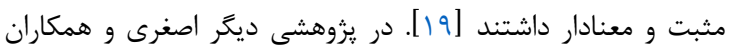

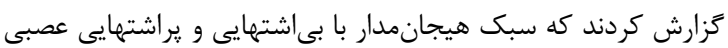

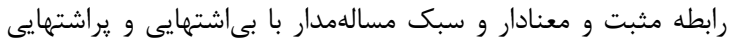

طبق نتايج جدول أ، در مدل اول ابتدا متغير راهبردهاى مثبت تنظيم

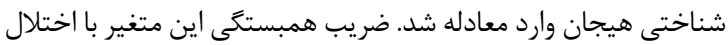

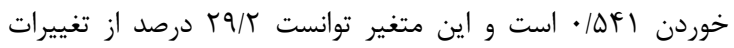

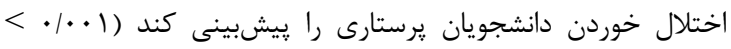

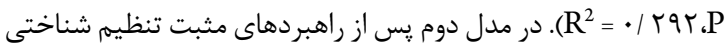

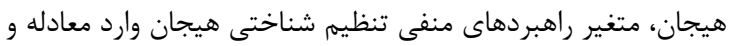

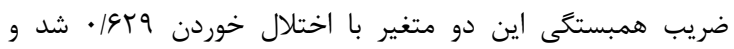

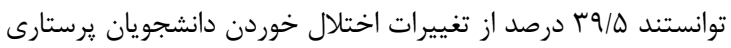

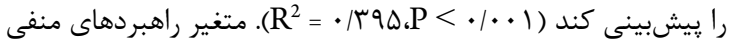

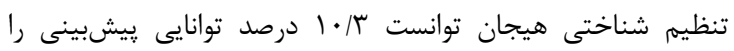

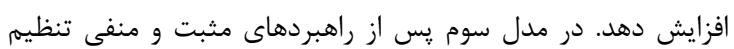

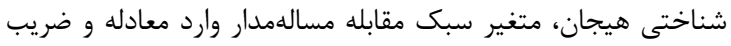

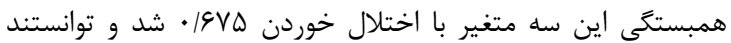

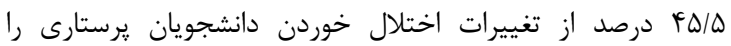

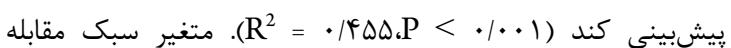

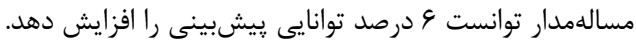

اين يزوهش با هدف پيشبينى اختلال خوردن دانشجويان يرستارى بر

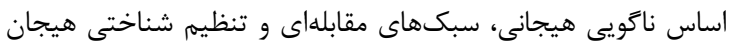

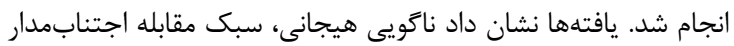

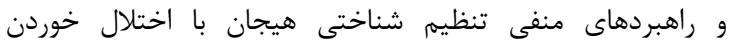

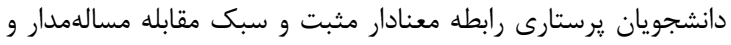

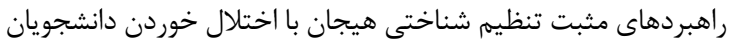

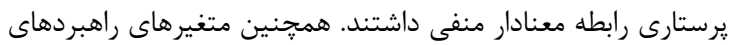

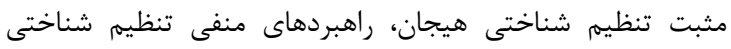

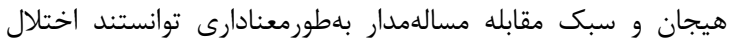

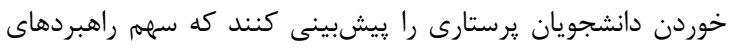

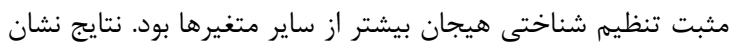

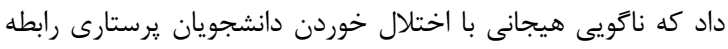

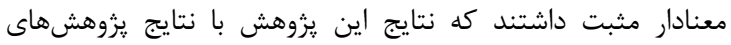

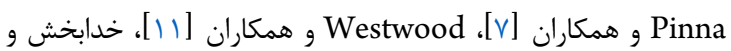


افزايش راهبردهاى منفى تنظيم شناختى هيجان ميزان اختلال خوردن

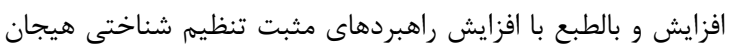

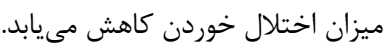

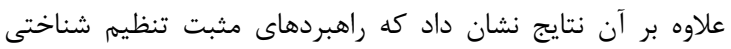

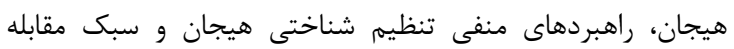

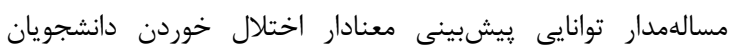

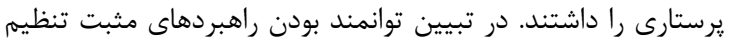

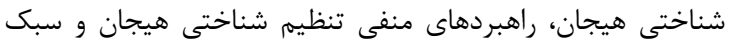

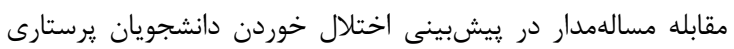

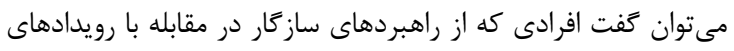

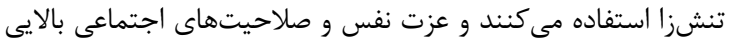

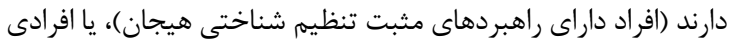

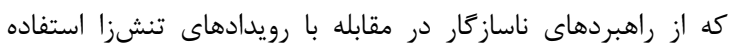

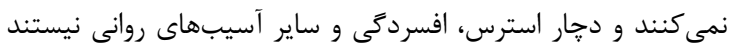

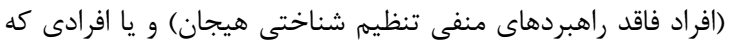

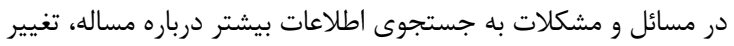

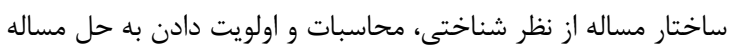

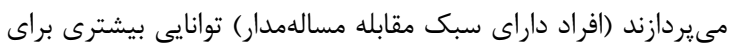

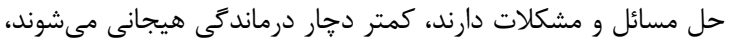

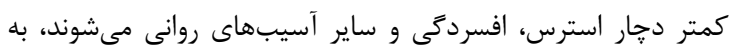

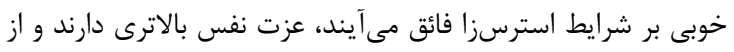

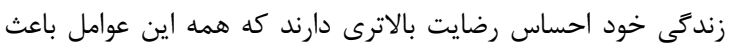

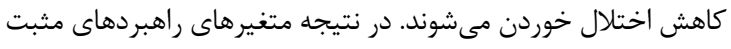

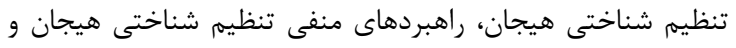

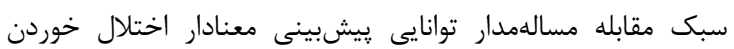
دانشجويان يرستارى را دارند.

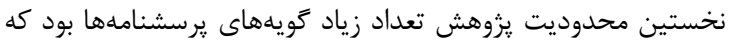

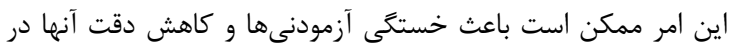

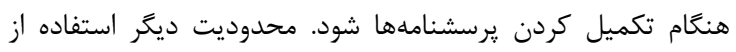

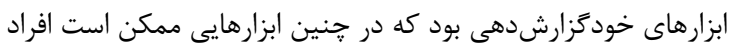

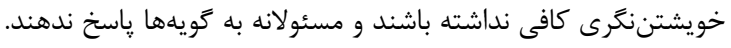

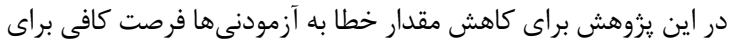

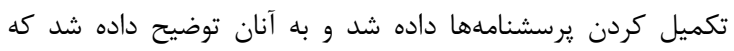

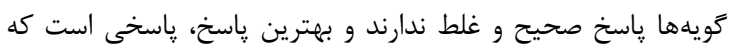

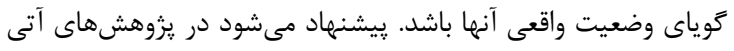

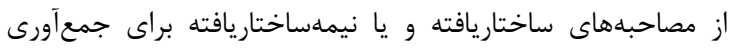

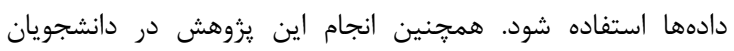

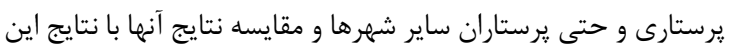

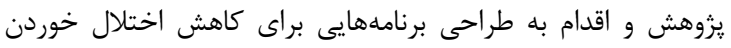

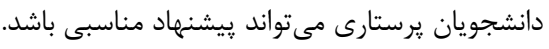

\section{نتيجه تيرى}

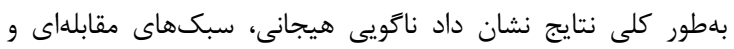

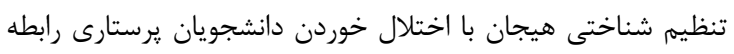

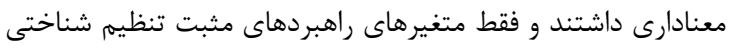
هيجان، راهبردهاى منفى تنظيم شناختى هيجان و و سبك مقان مقابله

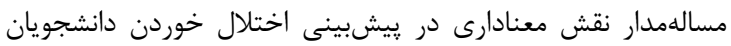

عصبى رابطه منفى و معنادار داشت [•r]]. در تبيين اين يافتهها بر

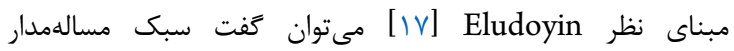

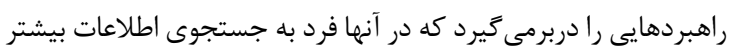

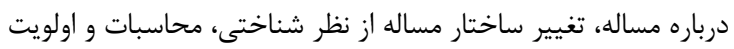

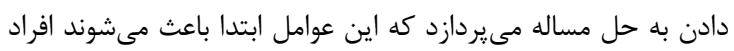

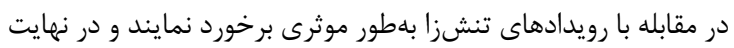

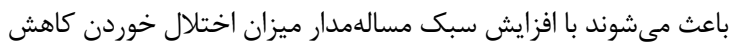

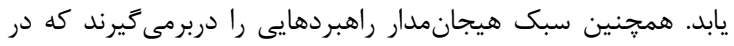

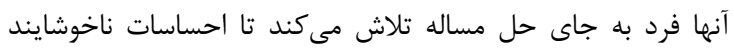

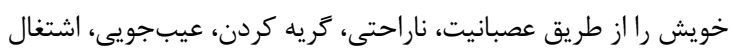

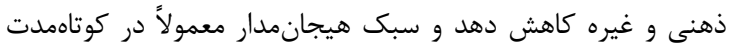

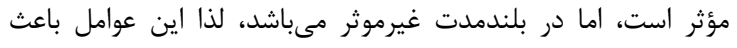

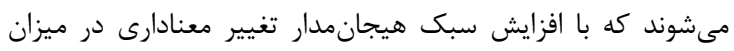

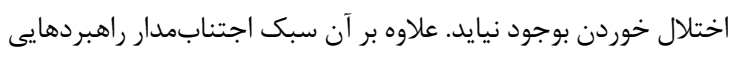

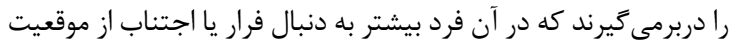

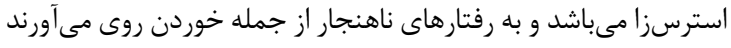

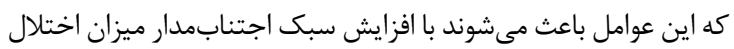
خوردن افزايش يابد. نتايج ديكر نشان داد كه راهبردهاى مثبت تنظيم شناختى هيجان بان إنان

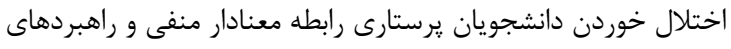

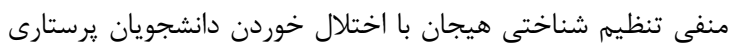

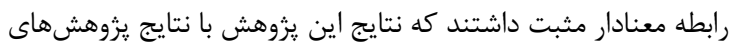
Micanti

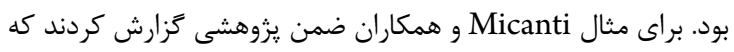
بين مشكلات تنظيم هيجان و اختلال خوردن رابطه معنادار مستقيم كردار

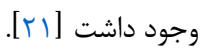

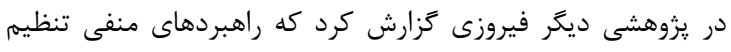

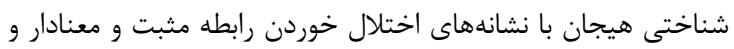

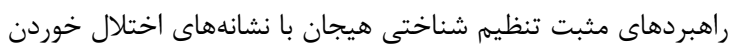

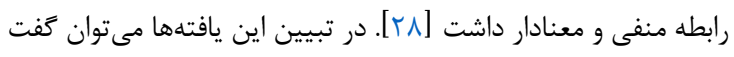

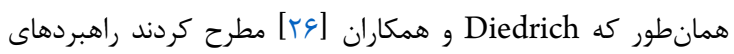

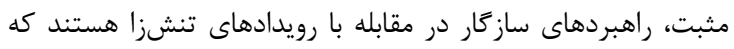

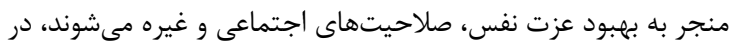

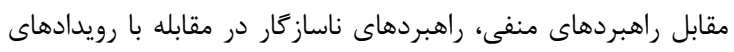

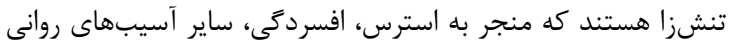

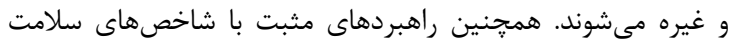

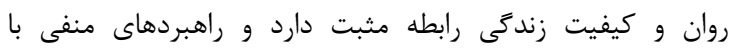

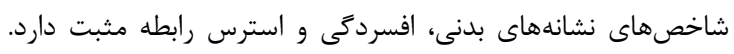

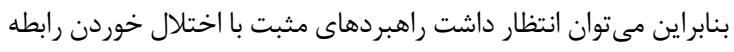

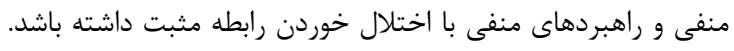

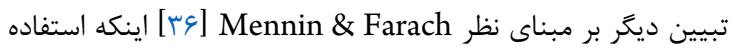

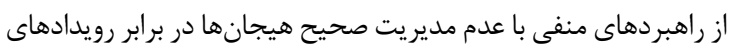

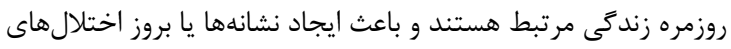

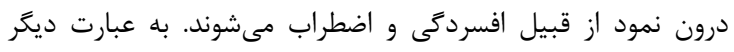

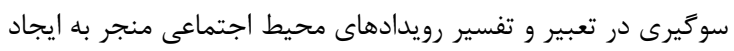

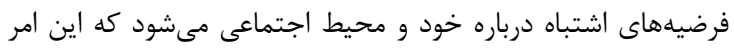

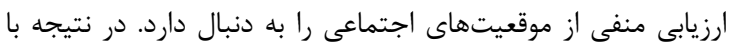




\section{سياسگزارى}

در يايان يزوهشكران از همه مسئولان و دانشجويان برستارى دانشكاه

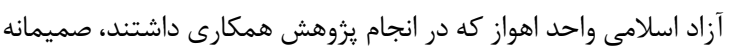

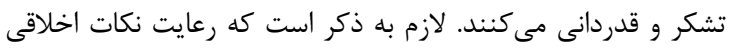

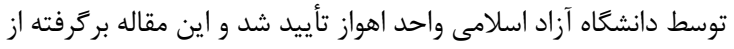

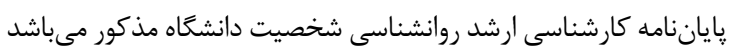

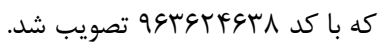
تضاد منافع

براى نويسند

\section{References}

1. Nicholls R, Perry L, Duffield C, Gallagher R, Pierce H. Barriers and facilitators to healthy eating for nurses in the workplace: an integrative review. J Adv Nurs. 2017;73(5):1051-65. doi: 10.1111/jan.13185 pmid: 27732741

2. Boisseau CL. Identification and management of eating disorders in gynecology: menstrual health as an underutilized screening tool. Am J Obstet Gynecol. 2016;215(5):572-8. doi: 10.1016/j.ajog.2016.07.009 pmid: 27422054

3. Goldschmidt AB, Wall MM, Choo TJ, Evans EW, Jelalian E, Larson N, et al. Fifteen-year Weight and Disordered Eating Patterns Among Community-based Adolescents. Am J Prev Med. 2018;54(1):e21-e9. doi: 10.1016/j.amepre.2017.09.005 pmid: 29132950

4. Moessner M, Fassnacht DB, Bauer S. Online assessment of eating disorders: the clinical and research inventory for eating disorders (CR-EAT). Ment Health Prev. 2015;3(4):170-7. doi: 10.1016/j.mhp.2015.08.001

5. Cook B, Leininger $\mathrm{L}$. The ethics of exercise in eating disorders: Can an ethical principles approach guide the next generation of research and clinical practice? J Sport Health Sci. 2017;6(3):295-8. doi: 10.1016/j.jshs.2017.03.004 pmid: 30356587

6. Cicmil N, Eli K. Body image among eating disorder patients with disabilities: a review of published case studies. Body Image. 2014;11(3):266-74. doi: 10.1016/j.bodyim.2014.04.001 pmid: 24958662

7. Pinna F, Sanna L, Carpiniello B. Alexithymia in eating disorders: therapeutic implications. Psychol Res Behav Manag. 2015;8:1-15. doi: 10.2147/PRBM.S52656 pmid: 25565909

8. Serafini G, Gonda X, Pompili M, Rihmer Z, Amore M, Engel-Yeger B. The relationship between sensory processing patterns, alexithymia, traumatic childhood experiences, and quality of life among patients with unipolar and bipolar disorders. Child Abuse Negl. 2016;62:39-50. doi: 10.1016/j.chiabu.2016.09.013 pmid: 27792883

9. Loas G, Baelde O, Verrier A. Relationship between alexithymia and dependent personality disorder: a dimensional analysis. Psychiatry Res. 2015;225(3):484-8. doi: 10.1016/j.psychres.2014.11.062 pmid: 25529260

10. DiStefano RA, Koven NS. Dysfunctional emotion processing may explain visual memory deficits in alexithymia. Pers Individ Differ 2012;52(5):611-5.

11. Westwood H, Kerr-Gaffney J, Stahl D, Tchanturia K. Alexithymia in eating disorders: Systematic review and meta-analyses of studies using the Toronto Alexithymia

$$
\begin{aligned}
& \text { يرستارى داشتند. بنابراين نتايج حاكى از اهميت راهبردهاى مثبت و }
\end{aligned}
$$

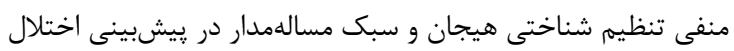

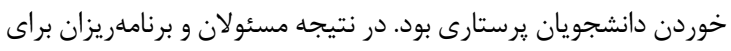

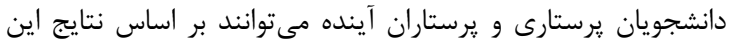

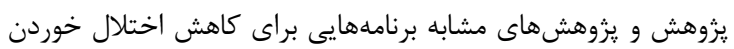

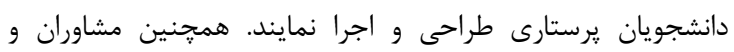

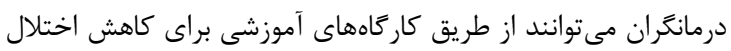

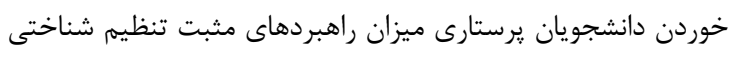

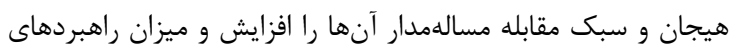

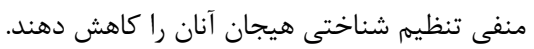

Scale. J Psychosom Res. 2017;99:66-81. doi: 10.1016/j.jpsychores.2017.06.007 pmid: 28712432

12. Khodabakhsh MR, Kiani F. The mediator role of emotion regulation difficulties in relationship between alexithymia and disordered eating behaviors among students AllamehTabataba'i University, Iran. Qom Univ Med Sci J. 2016;10(2):44-51.

13. Khodabakhsh M, Kiani F. The role of depressive symptoms and alexithymia in predicting disordered eating behavior among female students. J Commun Health. 2014;8(3):27-36.

14. Wonderlich-Tierney AL, Vander Wal JS. The effects of social support and coping on the relationship between social anxiety and eating disorders. Eat Behav. 2010;11(2):85-91. doi: 10.1016/j.eatbeh.2009.10.002 pmid: 20188291

15. Thompson NJ, Fiorillo D, Rothbaum BO, Ressler KJ, Michopoulos V. Coping strategies as mediators in relation to resilience and posttraumatic stress disorder. J Affect Disord. 2018;225:153-9. doi: 10.1016/j.jad.2017.08.049 pmid: 28837948

16. Alcala-Herrera V, Marvan ML. Early menarche, depressive symptoms, and coping strategies. J Adolesc. 2014;37(6):905-13.

10.1016/j.adolescence.2014.06.007 pmid: 25019174

17. Eludoyin OM. Assessment of daytime physiologic comfort, its perception and coping strategies among people in tertiary institutions in Nigeria. Weather Climate Extr. 2015;10(2):70-84.

18. Kirsch AC. Examining the Moderating Role of Specific Coping Strategies on the Relationship between Body Image and Eating Disorders in College-Age Women. Chicago: Loyola University; 2014.

19. Kachooei M, Ashtiani A, Allahyari A. The Role of coping styles and personality traits in eating-disordered behaviors. Health Psychol. 2015;4(13):62-73.

20. Asghari F, Ghasemi Jobaneh R, Yousefi N, Saadat S, Rafiei Gazani F. Role of perceived stress and coping styles on the eating disorders of high school students of Rasht city in 2013. Commun Health J. 2014;8(2):28-38.

21. Micanti F, Iasevoli F, Cucciniello C, Costabile R, Loiarro $\mathrm{G}$, Pecoraro G, et al. The relationship between emotional regulation and eating behaviour: a multidimensional analysis of obesity psychopathology. Eat Weight Disord. 2017;22(1):105-15. doi: 10.1007/s40519-016-0275-7 pmid: 27068173

22. Yousefi R, Asghari H, Toghyani E. Comparison of early maladaptive schemas and cognitive emotion regulation in 
cardiac patients and normal individuals. J Zanjan Univ Med Sci. 2017;24(107):130-43.

23. Zlomke KR, Hahn KS. Cognitive emotion regulation strategies: Gender differences and associations to worry. Pers Individ Differ 2010;48(4):408-13. doi: 10.1016/j.paid.2009.11.007

24. Baseri LD, Bozorgi ZD. Effectiveness of group therapy based on acceptance and commitment on cognitive emotion regulation and alexithymia of patients with type 2 diabetes. Iran J Psychiat Nurs. 2017;5(1):7-14. doi: 10.21859/ijpn-05012

25. Sakakibara R. Does cognitive appraisal moderate the effects of cognitive emotion regulation strategies? A shortterm longitudinal study. Pers Individ Differ 2016(101):511-2. doi: 10.1016/j.paid.2016.05.284

26. Diedrich A, Hofmann SG, Cuijpers P, Berking M. Selfcompassion enhances the efficacy of explicit cognitive reappraisal as an emotion regulation strategy in individuals with major depressive disorder. Behav Res Ther. 2016;82:1-10. doi: 10.1016/j.brat.2016.04.003 pmid: 27152671

27. Mac Vie JD. The Association between Emotion Regulation Strategies and Symptoms of Binge Eating Disorder. California California Lutheran University; 2016.

28. Firoozi A. Relationship between attachment styles, cognitive emotion regulation strategies, concerns about body image with eating disorder symptoms in students of Ahvaz Jundishapur University of medical sciences. Iran: Shahid Chamran University of Ahvaz; 2012.
29. Berthoz S, Perdereau F, Godart N, Corcos M, Haviland MG. Observer- and self-rated alexithymia in eating disorder patients: levels and correspondence among three measures. J Psychosom Res. 2007;62(3):341-7. doi: 10.1016/j.jpsychores.2006.10.008 pmid: 17324685

30. Shayeghian Z, Vafaae M. The evaluation of psychometric properties of eating disorder inventory (EDI). QJ Psychol Stud. 2010;6(2):9-23.

31. Aydin A. A comparison of the alexithymia, selfcompassion and humour haracteristics of the parents with mentally disabled and autistic children. Soc Behav Sci. 2015;174:720-9. doi: 10.1016/j.sbspro.2015.01.607

32. Besharat MA. Reliability and factorial validity of a Farsi version of the 20 -item Toronto Alexithymia Scale with a sample of Iranian students. Psychol Rep. 2007;101(1):209-20. doi: 10.2466/pr0.101.1.209-220 pmid: 17958129

33. Ghoreyshi Rad F. Validation of Endler \& Parker coping scale of stressful situations. J Behav Sci. 2010;4(1):1-7.

34. Garnefski N, Kraaij V, Spinhoven P. Negative life events, cognitive emotion regulation and emotional problems. Pers Individ Differ 2001;30(8):1311-27. doi: 10.1016/S0191-8869(00)00113-6

35. Sharifibastan F. The role of cognitive emotion regulation and positive and negative affect in resiliency of women with breast cancer. Iran J Psychiatry Nurs. 2016;4(2):3849. doi: 10.21859/ijpn-04025

36. Mennin D, Farach F. Emotion and evolving treatments for adult psychopathology. Clin Psychol Sci Pract. 2007;14(4):329-52. doi: 10.1111/j.14682850.2007.00094.x 